\title{
Island songbirds as windows into evolution in small populations
}

\author{
Thibault Leroy, ${ }^{1,2, *}$ Marjolaine Rousselle, ${ }^{1,3}$ Marie-Ka Tilak, ${ }^{1}$ Aude E. Caizergues, ${ }^{1,4}$ Céline Scornavacca, ${ }^{1}$ \\ María Recuerda, ${ }^{5}$ Jérôme Fuchs, ${ }^{6}$ Juan Carlos Illera, ${ }^{7}$ Dawie H. De Swardt, ${ }^{8}$ Guillermo Blanco, ${ }^{9}$ Christophe Thébaud, ${ }^{10}$ \\ Borja Milá, ${ }^{5}$ and Benoit Nabholz ${ }^{1,11,12, *}$ \\ ${ }^{1}$ ISEM, Université de Montpellier, CNRS, IRD, EPHE, Montpellier, France \\ ${ }^{2}$ Department of Botany \& Biodiversity Research, University of Vienna, Vienna, Austria \\ ${ }^{3}$ Bioinformatics Research Centre, Aarhus University, C.F. Møllers Alle 8, 8000 Aarhus, Denmark \\ ${ }^{4}$ CEFE, Université de Montpellier, CNRS, University Paul Valéry Montpellier 3, EPHE, IRD, Montpellier, France \\ ${ }^{5}$ National Museum of Natural Sciences (MNCN), Spanish National Research Council (CSIC), Madrid, Spain \\ 'Institut de Systématique, Evolution, Biodiversité (ISYEB), Muséum National d'Histoire Naturelle, CNRS, Sorbonne Université, EPHE, \\ Université des Antilles, CP51, 57 rue Cuvier, 75005 Paris, France \\ ${ }^{7}$ Biodiversity Research Unit (UO-CSIC-PA), Oviedo University, 33600 Mieres, Asturias, Spain \\ ${ }^{8}$ Department of Ornithology, National Museum, Bloemfontein, South Africa \\ ${ }^{9}$ Department of Evolutionary Ecology, National Museum of Natural Sciences (MNCN), Spanish National Research Council (CSIC), Madrid, \\ Spain \\ 10Laboratoire Evolution et Diversité Biologique (EDB), UMR 5174 CNRS - Université Paul Sabatier - IRD, Toulouse, France \\ ${ }^{11}$ Institut Universitaire de France (IUF), Paris, France \\ ${ }^{12}$ Lead contact \\ *Correspondence: thibault.leroy@univie.ac.at (T.L.), benoit.nabholz@umontpellier.fr (B.N.) \\ https://doi.org/10.1016/j.cub.2020.12.040
}

\section{SUMMARY}

Due to their limited ranges and inherent isolation, island species have long been recognized as crucial systems for tackling a range of evolutionary questions, including in the early study of speciation. ${ }^{1,2}$ Such species have been less studied in the understanding of the evolutionary forces driving DNA sequence evolution. Island species usually have lower census population sizes $(N)$ than continental species and, supposedly, lower effective population sizes $(\mathrm{Ne})$. Given that both the rates of change caused by genetic drift and by selection are dependent upon $\mathrm{Ne}$, island species are theoretically expected to exhibit (1) lower genetic diversity, (2) less effective natural selection against slightly deleterious mutations, ${ }^{3,4}$ and (3) a lower rate of adaptive evolution. ${ }^{5-8}$ Here, we have used a large set of newly sequenced and published whole-genome sequences of Passerida species (14 insular and 11 continental) to test these predictions. We confirm that island species exhibit lower census size and $\mathrm{Ne}$, supporting the hypothesis that the smaller area available on islands constrains the upper bound of $\mathrm{Ne}$. In the insular species, we find lower nucleotide diversity in coding regions, higher ratios of non-synonymous to synonymous polymorphisms, and lower adaptive substitution rates. Our results provide robust evidence that the lower $\mathrm{Ne}$ experienced by island species has affected both the ability of natural selection to efficiently remove weakly deleterious mutations and also the adaptive potential of island species, therefore providing considerable empirical support for the nearly neutral theory. We discuss the implications for both evolutionary and conservation biology.

\section{RESULTS}

To assemble our dataset, we used population-level sequencing data (Table 1) from 25 passerine bird species or subspecies, consisting of 14 insular and 11 continental, with a total of 295 individual whole-genome sequences (89 newly sequenced). All species belong to the Passerida lineage, a species-rich clade of songbirds with fairly similar life-history traits. Our dataset includes at least 4 independent continental-island transitions that occurred across the songbird phylogeny (Figure S1) enabling us to efficiently account for phylogenetic structure in all statistical tests reported below (phylogenetic generalized least square [PGLS]; see also Table S1 for additional tests).
Do island species exhibit genomic signatures consistent with low $\mathrm{Ne}$ ?

Past effective population sizes were inferred using the pairwise sequentially Markovian coalescent (PSMC) approach for one randomly selected individual from each species (Figure S2) and were then averaged over the last one million years. The analyses confirmed that island species exhibit a significantly lower mean $\mathrm{Ne}$ than continental species over the last one million years (mean $\mathrm{Ne}=362,456$ and 94,944 for continental and island species, respectively, Figure 1A; log-transformed Ne, PGLS $p=1.0 \times 10^{-4}$ ). Specifically, inferred mean $N e$ values over the last million years range from $6.1 \times 10^{4}$ for the Tenerife blue chaffinch (Fringilla teydea) to $1.2 \times 10^{6}$ for a continental population of 


\begin{tabular}{|c|c|c|c|c|c|}
\hline & Species & Clade & Range & Individuals & Data \\
\hline 1 & Certhidea olivacea (S) & Darwin's finches & island & 5 & 9 \\
\hline 2 & Certhidea fusca (E) & Darwin's finches & island & 10 & 9 \\
\hline 3 & Certhidea fusca ( $L$ ) & Darwin's finches & island & 10 & 9 \\
\hline 4 & Platyspiza crassirostris (Z) & Darwin's finches & island & 5 & 9 \\
\hline 5 & Camarhynchus pallidus (Z) & Darwin's finches & island & 5 & 9 \\
\hline 6 & Pinaroloxias inornata (C) & Darwin's finches & island & 8 & 9 \\
\hline 7 & Geospiza difficilis $(P)$ & Darwin's finches & island & 10 & 9 \\
\hline 8 & Geospiza septentrionalis (W) & Darwin's finches & island & 8 & 9 \\
\hline 9 & Geospiza conirostris (E) & Darwin's finches & island & 10 & 9 \\
\hline 10 & Ficedula albicollis & Ficedula flycatchers & continental & 20 & 10 \\
\hline 11 & Ficedula hypoleuca & Ficedula flycatchers & continental & 20 & 10 \\
\hline 12 & Ficedula speculigera & Ficedula flycatchers & continental & 20 & 10 \\
\hline 13 & Ficedula semitorquata & Ficedula flycatchers & continental & 20 & 10 \\
\hline \multirow[t]{3}{*}{14} & Zosterops borbonicus & white-eyes & island & 6 & 11 \\
\hline & & & & 1 & 12 \\
\hline & & & & 18 & this study \\
\hline 15 & Zosterops olivaceus & white-eyes & island & 15 & this study \\
\hline 16 & Zosterops mauritianus & white-eyes & island & 9 & this study \\
\hline 17 & Zosterops pallidus & white-eyes & continental & 2 & this study \\
\hline 18 & Zosterops virens & white-eyes & continental & 11 & this study \\
\hline 19 & Fringilla coelebs & chaffinches & continental & 9 & this study \\
\hline 20 & Fringilla coelebs palmae & chaffinches & island & 15 & this study \\
\hline 21 & Fringilla teydea & chaffinches & island & 10 & this study \\
\hline 22 & $\begin{array}{l}\text { Taeniopygia guttata } \\
\text { castanotis }\end{array}$ & Estrildidae & continental & 19 & 13 \\
\hline 23 & $\begin{array}{l}\text { Poephila acuticauda } \\
\text { acuticauda }\end{array}$ & Estrildidae & continental & 10 & 13 \\
\hline 24 & Parus major & Paridae & continental & 10 & 14 \\
\hline 25 & Phylloscopus trochilus & Phylloscopidae & continental & 9 & 15 \\
\hline
\end{tabular}

The abbreviation in parenthesis following Darwin's finches names indicates the island of origin (C, Coco; E, Española; L, San Cristobal; P, Pinta; S, Santiago; W, Wolf; Z, Santa Cruz). See also Methods S1.

the common chaffinch ( $F$. coelebs), representing an $\sim 20$ fold difference (Figure 1A; Table S2).

Such long-term differences in $\mathrm{Ne}$ between insular and continental species are expected to generate differences in nucleotide diversity levels, because genetic variation is determined by both mutation rate and effective population size. By estimating nucleotide diversity at synonymous $\left(\pi_{\mathrm{S}}\right)$ and at non-synonymous sites $\left(\pi_{N}\right)$, we find marked differences between island and continental species. Using 6,499 orthologous genes on average (range: $5,018-7,514$, among 8,253 orthogroups ${ }^{16}$ ), we find that $\pi_{\mathrm{S}}$ varies from $0.07 \%$ in the Tenerife blue chaffinch to $1.25 \%$ in the willow warbler (Phylloscopus trochilus), representing a 17-fold difference between these island and continental species (Table S2; Figure S3). Island species exhibit significantly lower mean $\pi_{\mathrm{S}}$ than continental species (mean $\pi_{\mathrm{S}}=0.59 \%$ and $0.18 \%$ for continental and island species, respectively, Figure 1B; PGLS, $p=5.29 \times 10^{-3}$ ).

In addition to strong evidence for lower $\mathrm{Ne}$ in island species, we also find lower census population sizes in the island species (island: 7 species, median: $1.1 \times 10^{4}$ [range: $6.2 \times 10^{2}-3.0 \times$
$10^{5}$ ]; continental: 6 species, median: $2.5 \times 10^{8}$ [range: $2.0 \times$ $10^{5}-5.7 \times 10^{8}$ ]; log-transformed census sizes, PGLS, $p=$ $8.29 \times 10^{-5}$ ). Furthermore, both $\log _{10}$-transformed current census population sizes and geographical range in square kilometers are positively correlated with $\pi_{\mathrm{S}}$ (Figures $2 \mathrm{~A}$ and $2 \mathrm{C}$; PGLS, $p<0.01$; Table S1). Taken all together, these results provide strong support for the view that long-term restrictions on census population sizes due to the limited surface area available to island species constrains the upper bound of effective population size.

\section{Are deleterious mutations segregating more in island species?}

Based on the nearly neutral theory of molecular evolution, the higher level of genetic drift associated with lower $\mathrm{Ne}$ is expected to contribute to an accumulation of slightly deleterious mutations in island species relative to their continental counterparts. Using the ratio of non-synonymous to synonymous mutations $\left(\pi_{N} / \pi_{S}\right)$ as a proxy for the proportion of these slightly deleterious mutations, we recover, on average, a $40 \%$ higher $\pi_{N} / \pi_{S}$ in island 
A

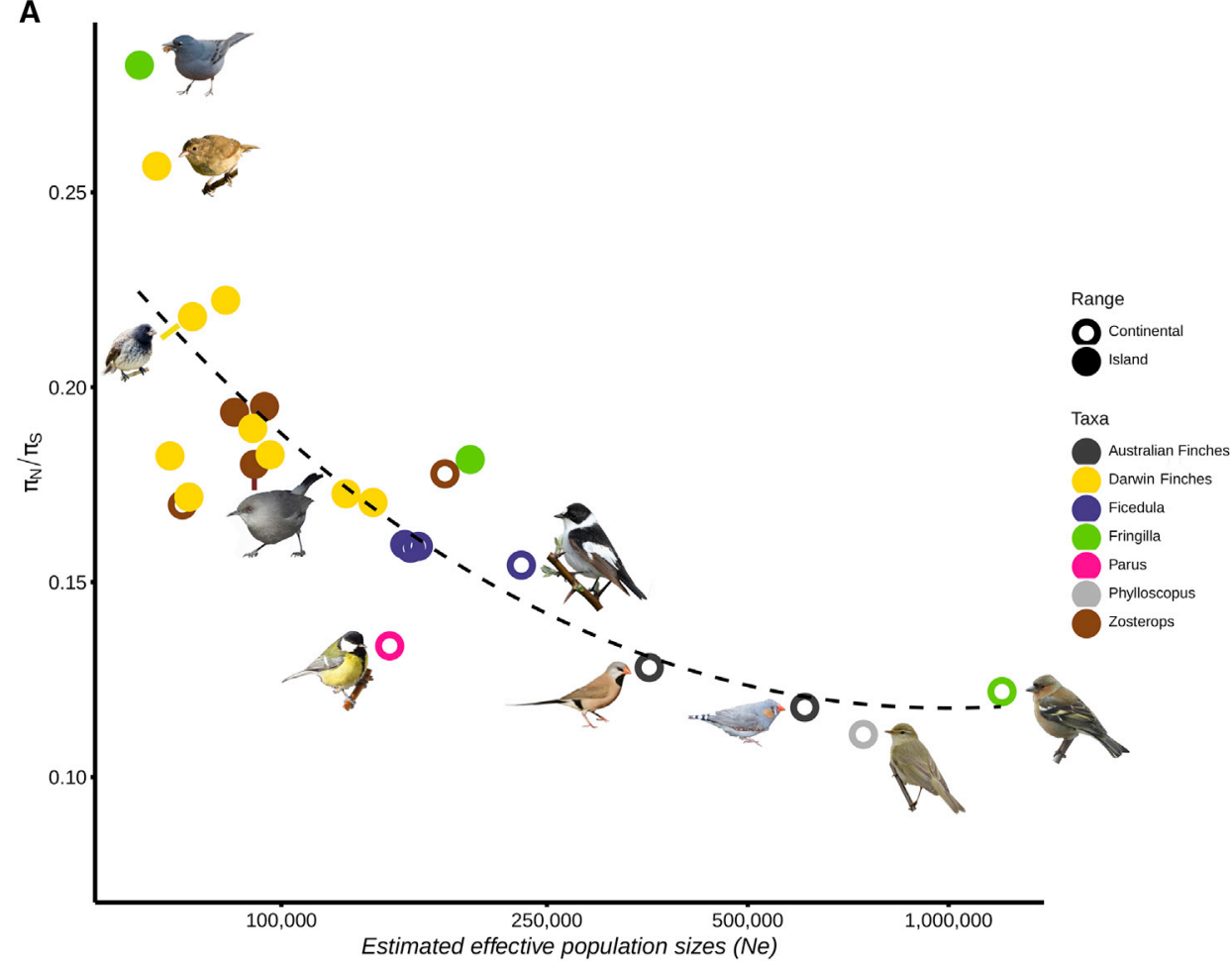

B
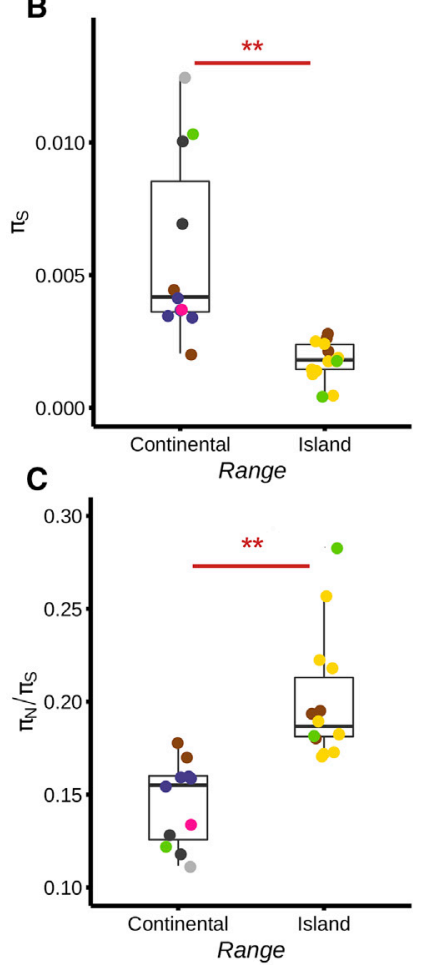

Figure 1. Island species as models for evolution in small effective population sizes

(A) Local polynomial regression (LOESS with span $=1.25)$ between the ratio of nonsynonymous to synonymous nucleotide diversity $\left(\pi_{\mathrm{N}} / \pi_{\mathrm{S}}\right)$ and the mean effective population sizes over the last million years (Ne), as inferred using PSMC (see Figure S3 for a log-log regression between $\pi_{N} / \pi_{S}$ and $\pi_{S}$ estimates, respectively).

(B and C) Variation in nucleotide diversity $\left(\pi_{\mathrm{S}}, \mathrm{B}\right)$ and $\pi_{N} / \pi_{\mathrm{S}}$ between island endemic and continental species (C). Red asterisks indicate significance of the PGLS test $\left({ }^{\star \star} p<0.01\right)$.

Photo credits: A. Chudý, F. Desmoulins, E. Giacone, G. Lasley, Lianaj, Y. Lyubchenko, B. Nabholz, J.D. Reynolds, K. Samodurov, A. Sarkisyan (iNaturalist.org); M. Gabrielli (personal communication). See also Figures S1-S3 and Tables S1 and S2.

species than in continental species (Figure 1C; mean $\pi_{N} / \pi_{S}=$ 0.145 and 0.201 for continental and island species, respectively, PGLS $\mathrm{p}=4.57 \times 10^{-3}$ ).

In addition, we find substantial within-genome variation in the accumulation of slightly deleterious mutations, as well as in the levels of nucleotide diversity, in such a way that $\pi_{S}$ and $\pi_{N} / \pi_{S}$ are, respectively, positively and negatively correlated to the GC content at the third codon position (GC3). GC3 provides a robust proxy of recombination rate in birds ${ }^{17,18}$ (Methods S1). By comparing sets of genes exhibiting the lowest and highest GC3, we found a more marked $\pi_{N} / \pi_{S}$ differences in genes exhibiting low GC3 $\left(\Delta\right.$ mean $_{\text {continental versus Island }}=0.107,95 \% \mathrm{Cl}$ : $0.077-0.194)$ than in those exhibiting high GC3 $(\Delta$ mean $=0.029$, 95\% Cl: $0.011-0.048)$. The stronger effect of recombination for island species is captured by the significant interaction between GC3 and insularity in the linear model: $\pi_{N} / \pi_{S}$ $\sim$ GC3+insularity+GC3:insularity $\left(R^{2}=0.72, p\right.$ value model $<2.2 \times 10^{-16}$, including $\mathrm{p}<2.2 \times 10^{-16}, 2.8 \times 10^{-10}$, and $1.33 \times 10^{-05}$ for GC3, insularity, and the interaction, respectively). These correlations are found to be stronger in island species relative to their continental counterparts, with a particularly pronounced difference in $\pi_{N} / \pi_{S}$ in genes exhibiting a low GC3. These results are robust to a control for GC-biased gene conversion
(Methods S1). Recombination limits genetic interactions between selected mutations and can therefore improve the efficiency of selection. ${ }^{19,20}$ These results suggest that the intensity of the differences between island and continental species in the effectiveness of purifying selection relies heavily on the local genomic context.

We found strong negative correlations between (1) $\pi_{N} / \pi_{S}$ and the $\log _{10}$-transformed $\mathrm{Ne}$ averaged over the last one million years (Figure 1A; PGLS, $p=1.0 \times 10^{-4}$ ) and (2) the nontransformed $\pi_{N} / \pi_{S}$ and $\pi_{S}$ values (PGLS, $p=4.85 \times 10^{-5}$ ). $\log _{10}$-transformed current census population sizes, as well as geographical range sizes, significantly correlate with $\pi_{N} / \pi_{S}$ (Figure 2). In contrast, the IUCN red list assessments have no effect on $\pi_{N} / \pi_{S}$ or $\pi_{S}$ (Table S1; PGLS p >> 0.05). Taken together, our results provide strong empirical evidence that differences in census population sizes between island and continental species translate into differences in $\mathrm{Ne}$, and that these differences have a marked influence on genetic diversity and the efficiency of natural selection. These findings fit remarkably well with the expectation from the nearly neutral theory.

Do insular species show lower adaptive potential? Theory predicts that lower $\mathrm{Ne}$ in island species should lead to a lower rate of adaptive substitutions than in continental species, if 
A
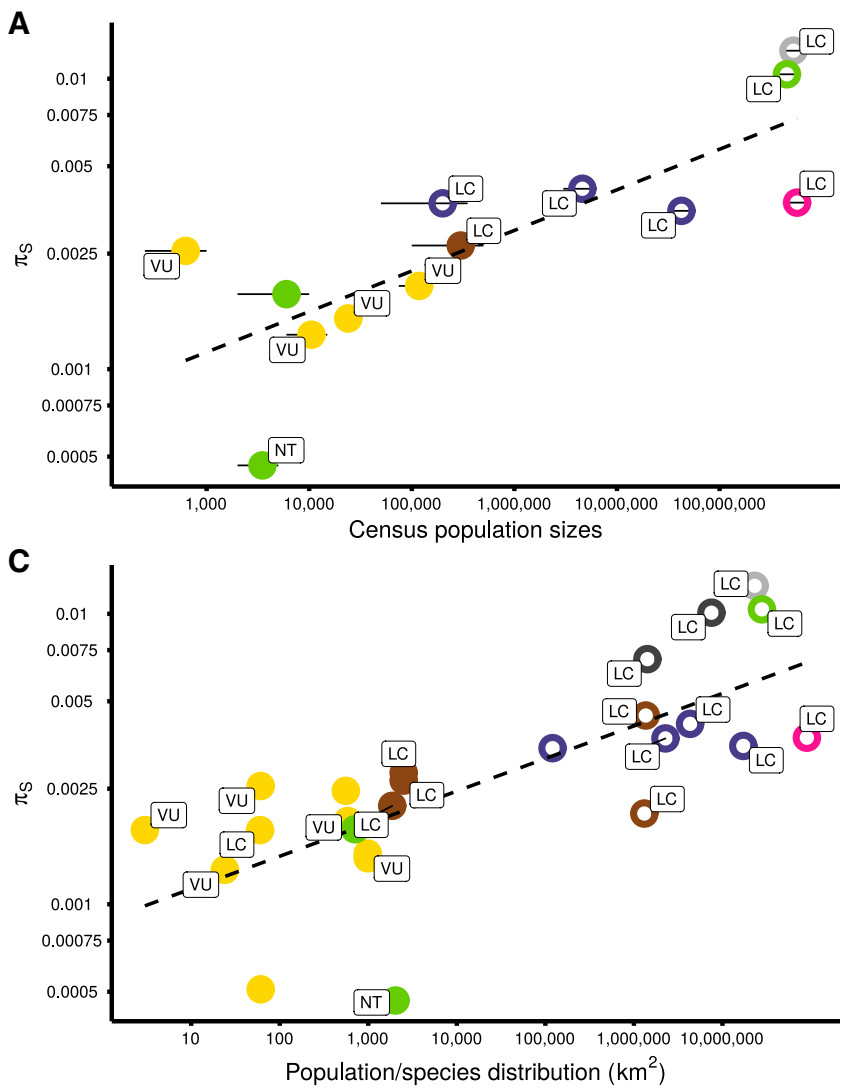
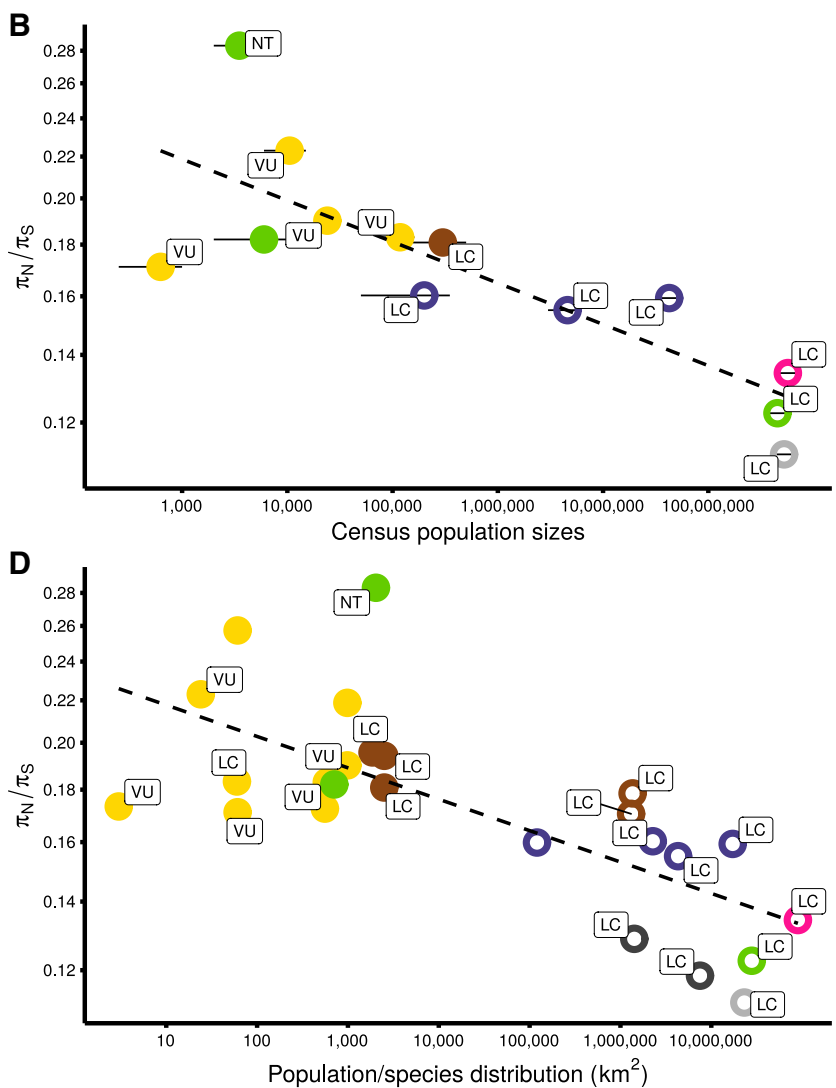

Figure 2. Ecological-evolutionary correlations based on the variables investigated in this study $\pi_{\mathrm{S}}$ and $\pi_{\mathrm{N}} / \pi_{\mathrm{S}}$ are used as proxies of $\mathrm{Ne}$ and the efficiency of natural selection to remove deleterious variants and are correlated with both the median estimates of the current census population sizes ( $A$ and $B$ ) and the geographical range sizes ( $C$ and $D)$. Both ecological and evolutionary parameters are log-transformed. Filled and open dots represent the island and the continental species, respectively (Figure 1 for details). Only the 13 species with estimates of the current census population sizes are included for the (A) and (B) (with ranges shown with a thin black line). Where known, the IUCN conservation status of the investigated species is indicated (LC, least concerned; NT, near threatened; VU, vulnerable). See also Figure S3 and Tables S1 and S2.

adaptation is limited by the supply of new mutations ${ }^{8}$ and/or if slightly advantageous mutations become effectively neutral in low $\mathrm{Ne}$ species. ${ }^{6}$ For taxa with at least two species (i.e., all except Parus and Phylloscopus), we used the maximum likelihood method implemented in Grapes ${ }^{21}$ to estimate non-adaptive rate of substitution ( $\left.\omega_{\mathrm{NA}}\right)$ and adaptive rate of substitution $\left(\omega_{A}\right)$ with $\omega$ (i.e., $d_{N} / d_{S}$ ) being the sum of $\omega_{N A}+\omega_{A}$. No significant difference in $\omega$ was observed between island and continental species $(\omega$ island $=0.194$ and $\omega$ continental $=0.187$ ). By contrast, island species showed a higher $\omega_{\mathrm{NA}}\left(\Delta\right.$ mean $_{\text {continental versus Island }}=$ $0.063)$ and a lower $\omega_{\mathrm{A}}(\Delta$ mean continental versus Island $=0.056)($ Figure 3; see Figure $S 4$ for $\alpha$ estimates) than continental counterparts. However, these differences are only significant for tests that did not explicitly take phylogenetic structure into account (PGLS, $p=0.257$ and $p=0.237$; non-PGLS, $p=0.014$ and $p=$ 0.002 for $\omega_{\mathrm{A}}$ and $\omega_{\mathrm{NA}}$, respectively; Table S1), and therefore they should be interpreted with caution.

We found that $\omega_{\mathrm{A}}$ was positively correlated with $\log _{10}$-transformed $\pi_{S}$ (PGLS, $p=0.029$; Figure $3 A$ ) and negatively correlated with the $\log _{10}$-transformed $\pi_{N} / \pi_{S}$ (PGLS, $p=0.034$; Table $\mathrm{S} 1)$. Reciprocally, $\omega_{\mathrm{NA}}$ is significantly negatively correlated with log10-transformed $\pi_{\mathrm{S}}$ (PGLS, $\mathrm{p}=0.020$; Figure $3 \mathrm{~B}$ ) and positively with $\log _{10}$-transformed $\pi_{N} / \pi_{S}$ (PGLS, $p=0.025$; Figure S4).

Overall, our analysis suggests that a lower Ne doubly affects island species relative to continental species, because (1) relatively fewer adaptive mutations can reach fixation, and (2) the lower efficiency of natural selection allows a greater proportion of weakly deleterious variants to reach fixation in insular species.

\section{DISCUSSION}

Our analysis of whole-genome resequencing data has allowed us to find lower nucleotide diversity, a higher frequency of slightly deleterious mutations, and lower adaptive substitution rates in the island species than in the continental ones. These results provide important insights for evolutionary biology, and they also have major implications for the conservation of species with small populations.

Island species as models for studying the evolutionary consequences of small $\mathrm{Ne}$

The smaller land area available on oceanic islands should constrain the upper bound of both census and effective 

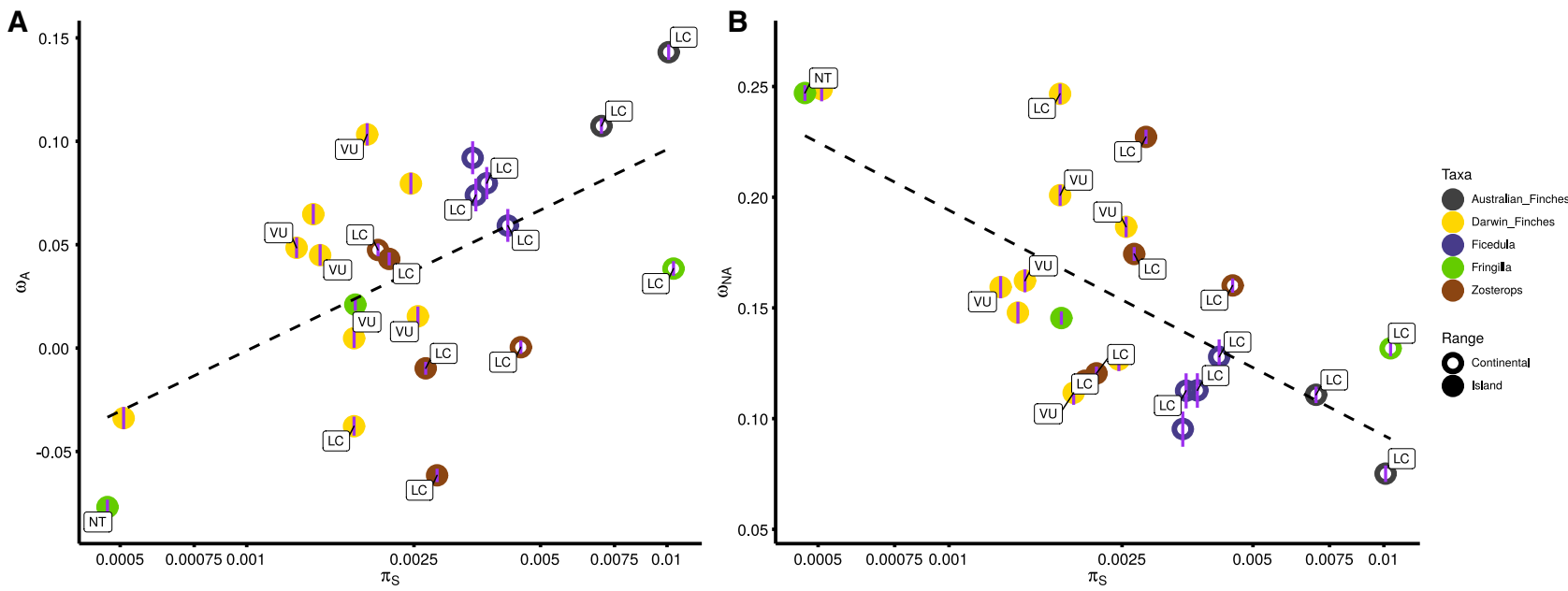

Figure 3. Relationships between adaptive and non-adaptive susbtitution rates and synonymous genetic diversity Proportion of adaptive (A) and non-adaptive (B) substitutions along the neutral genetic diversity gradient $\left(\pi_{\mathrm{S}}\right)$ as estimated by comparing the observed and the expected $d_{N} / d_{S}$ under near neutrality assuming the polymorphism data using the DFE- $\alpha$ method $\left(\alpha\right.$ shown Figure $S 4 ;$ with $\left.\omega_{A}=\alpha\left(d_{N} / d_{S}\right) \& \omega_{N A}=(1-\alpha)\left(d_{N} / d_{S}\right)\right)$. Estimates were performed using all sites and the GammaExpo model. Error bars (purple line) represent the $95 \%$ confidence intervals of each estimate under this model. Where known, the IUCN conservation status of the investigated species is indicated (LC, least concerned; NT, near threatened; VU, vulnerable). See also Table S1.

population sizes of insular species, to such an extent that demography affects the ability of purifying selection to remove weakly deleterious mutations. Our results are largely consistent with this general hypothesis and suggest that contemporary census sizes provide information on long-term $\mathrm{Ne}$ (but see also Díez-Del-Molino et al. ${ }^{22}$ and Peart et al. ${ }^{23}$ ). For most population genomic estimates we investigated, including $\pi_{S}, \pi_{N} / \pi_{S}$ and PSMC-inferred $\mathrm{Ne}$, we observed significant differences between continental and island species that are consistent with theoretical expectations.

Previous taxon-specific studies have reported low $\mathrm{Ne}$ in a diverse range of island organisms (e.g., giant Galápagos tortoises, ${ }^{24}$ woolly mammoths, ${ }^{25}$ island foxes, ${ }^{26,27}$ and Corvus ${ }^{28}$ ). Therefore, it is very likely that island species predominantly exhibit lower Ne than their more abundant, broadly distributed, mainland relatives, and this pattern may not be restricted to some specific animal clades such as birds or mammals but may also be true for a large range of taxa (e.g., Hamabata et al. ${ }^{29}$ for plants). More broadly, this result opens up new opportunities for using island species as models to understand the impact of $\mathrm{Ne}$ on genome evolution in natural populations, including genome size, or of natural selection on non-coding genomic regions.

\section{Broad support for the nearly neutral theory of molecular evolution}

Fifty years after the introduction of the neutral theory of molecular evolution by Kimura ${ }^{30}$ and King and Jukes, ${ }^{31}$ and, after being extended into the nearly neutral theory, ${ }^{4}$ the neutralist-selectionist controversy remains one of the sharpest and most polarized debates in biology. Based on our large genome-scale empirical data, our results match theoretical expectations of the nearly neutral theory remarkably well. This is consistent with the strength of this theory in explaining patterns of DNA sequence evolution, allowing us to affirm that the nearly neutral theory is overwhelmingly supported by our dataset. Slightly deleterious mutations are frequent and become effectively neutral when the effect of genetic drift increases, as is typically observed in insular species.

Selective processes, including positive selection on beneficial alleles and background selection, play an important role in the sequence evolution of the investigated species but cannot be used to reject the theory as a whole. Empirical investigations found that the proportion of adaptive substitutions does not overall scale with $\mathrm{Ne}$ when distant taxa are considered all together (e.g., Galtier ${ }^{21}$ ), but taxa-specific investigations were able to find such a relationship, with a lower proportion of adaptive substitutions in species with a lower $\mathrm{Ne}$, as recently reported for several groups of animals. ${ }^{8}$ First, our analyses provide additional evidence for such a relationship in passerine birds. Second, we indeed observe that local recombination rates influence both local levels of nucleotide diversity and the number of deleterious mutations, which is consistent with heterogeneous landscapes of $\mathrm{Ne}$ throughout genomes. ${ }^{18}$ However, significant differences between island and continental species were similarly recovered in both lowly and highly recombining regions of the genome, supporting the claim that background selection does not fundamentally change the predictions that can be drawn from the theory.

\section{Ecological-evolutionary ties and perspectives}

At the macroevolutionary scale, strong correlations between lifehistory traits and both levels of polymorphism and ratios of nonsynonymous to synonymous mutations have been reported in the literature for both animals and plants, ${ }^{32-34}$ suggesting that determinants of genetic diversity are mostly ecologically driven. We found that nucleotide diversity scales positively with species range, which therefore suggests a gradual transition between species restricted to small islands and species widely distributed over continents. Recently, Peart et al. ${ }^{23}$ proposed that conservation priorities should be defined based on the ratio of census size 


\section{$\infty$ CellPress}

to $\mathrm{Ne}$. However, whether population genomic estimates of $\mathrm{Ne}$ are informative enough to assess conservation status is questionable. A general outcome is that animal species classified as threatened generally exhibit lower genetic diversity than those classified as non-threatened, including birds (at least at microsatellite $l o i^{35,36}$ ). Based on our whole-genome analyses, we can report no obvious contrast between the four island species classified as threatened (vulnerable status) and the species classified as non-threatened, neither for the levels of nucleotide diversity nor for their efficiency of natural selection (but see Brüniche-Olsen et al. ${ }^{37}$ ). Díez-del-Molino et al. ${ }^{22}$ were also unable to recover a significant effect of the IUCN assessment on the levels of nucleotide diversity in birds and mammals. Using 78 mammal species, Brüniche-Olsen et al. $^{38}$ only recovered this pattern when the animals' diets were explicitly taken into account. Consequently, it seems that we still have a long way to go toward precisely describing whether these genomic features are completely independent or are correlated to some extent with the current conservation status.

Another open question is whether population genomics can provide information so that short-term IUCN objectives can be extended over a longer time frame? Even if some island species accumulate slightly deleterious mutations, ${ }^{39}$ supposedly leading to increased maladaptation, we can question whether this burden of slightly deleterious mutations can lead to species extinction. This hypothesis holds true only if these deleterious mutations are neither purged nor opposed by compensatory or beneficial mutations. ${ }^{40}$ Remarkably, the four species classified as threatened are not those exhibiting the lowest proportion of adaptive substitutions (mean $\omega_{\mathrm{A}}=0.053$ compared to 0.036 for the 15 species with a least concern status). Recent macroevolutionary investigations, however, provide support for this increased risk of (1) being endangered depending on the time since the species colonized the island ${ }^{41}$ or (2) becoming extinct depending on the island size. ${ }^{42}$ Age-dependent processes such as ecological specialization were proposed, but the accumulation of deleterious mutations might explain this phenomenon as well. Rogers and Slatkin ${ }^{25}$ propose that, after a tipping point, this mutational meltdown might contribute to the ultimate steps in the road to extinction. Endemic island species therefore represent taxa of high interest in the evaluation of the long-term consequences of evolution under low effective population sizes.

\section{STAR $\star$ METHODS}

Detailed methods are provided in the online version of this paper and include the following:

- KEY RESOURCES TABLE

- RESOURCE AVAILABILITY

○ Lead contact

O Materials availability

O Data and code availability

- EXPERIMENTAL MODEL AND SUBJECT DETAILS

O Species included in the study

O Species distribution and IUCN red list status

- METHOD DETAILS

○ DNA extraction and sequencing (Zosterops and Fringilla species)
O Publicly available sequencing data

O Variant identification

- QUANTIFICATION AND STATISTICAL ANALYSIS

O Gene models \& orthology prediction

- Effective population size estimates

O Summary statistics of the polymorphic data

O Summary statistics of the divergence data

O Statistical analyses

\section{SUPPLEMENTAL INFORMATION}

Supplemental Information can be found online at https://doi.org/10.1016/j. cub.2020.12.040.

\section{ACKNOWLEDGMENTS}

This research was funded by the French ANR (BirdlslandGenomic project, ANR-14-CE02-0002). The analyses benefited from the Montpellier Bioinformatics Biodiversity (MBB) platform services, the genotoul bioinformatics platform Toulouse Midi-Pyrenees (Bioinfo Genotoul), and the Biogenouest BiRD core facility (Université de Nantes). We are grateful to Quentin Rougemont for providing feedback on a previous version of the manuscript, colleagues from the phylogeny and molecular evolution team at ISEM Montpellier for fruitful discussions throughout this project, and Neil McNair (University of Vienna) for proofreading the manuscript, as well as three anonymous reviewers for their constructive comments, which helped us to substantially improve the manuscript. A previous manuscript version also benefited from detailed discussions on Twitter; we would thus like to thank Graham Coop, Jenny James, Nicolas Rode, among others, for their helpful suggestions. The authors wish to thank all the farmers and private and national nature reserves where fieldwork was conducted. For South African samples, the handling and sampling protocols were approved by the Comité Cuvier (68-055 to J.F.). We are grateful to the provincial authorities in the Eastern Cape and Free State provinces of South Africa, and Eastern Cape Parks (Alan Southwood, Cathy Dreyer, Gavin Shaw, Sizwe Mkhulise) for granting permission to collect samples (permit numbers RA-190, CRO144/14CR, and 01-24158). We would also like to acknowledge the Percy FitzPatrick Institute (University of Cape Town), the National Museum Bloemfontein (Free State), and R.C.K. Bowie, P.-H. Fabre, E. Kolarova, and G. Oatley for help during field work and logistical support. For Reunion samples, we thank the Reunion National Park for granting us permission to conduct fieldwork in Pas de Bellecombe, Reunion, France and the field station of Marelongue, funded by the P.O.E., Reunion National Park and OSU Reunion, for logistical support. For Spanish samples, the Canary government gave permission to perform the sampling work to J.C.I. (permit 01-24158). J.C.I. was funded by the Spanish Ministry of Science, Innovation and Universities (Ref.: PGC2018-097575-B-100) and by a GRUPIN research grant from the Regional Government of Asturias (Ref.: IDI/2018/000151). B.M. and M.R. were partly funded by the Spanish Ministry of Science and Innovation (grant PGC2018-098897-B-100), and M.R. was supported by a doctoral fellowship from the Spanish Ministry of Education, Culture, and Sport (FPU16/05724). This is publication ISEM 2020-321.

\section{AUTHOR CONTRIBUTIONS}

T.L. designed the research, conducted the analyses, and wrote the paper. B.N. designed the research, contributed to the analyses, supervised the work, and wrote the paper. M.R. contributed to the analysis of the divergence data. M.-K.T. performed the wet lab work. A.E.C. performed preliminary investigations using the publicly available data and performed the analysis to identify the Darwin's finch populations and species to consider for the paper. C.S. conducted the analyses of orthologous assignation. M.R., G.B., and B.M. provided the sequence data of Fringilla coelebs species and the reference Fringilla genome. J.C.I. provided the Fringilla samples used in this study. D.H.D.S. and J.F. helped in field work to collect mainland and island Zosterops samples. C.T. and B.M. helped during field work in Reunion and revised the manuscript. All the authors read and approved the manuscript. 


\section{DECLARATION OF INTERESTS}

The authors declare no competing interests.

Received: April 14, 2020

Revised: October 12, 2020

Accepted: December 23, 2020

Published: January 20, 2021

\section{REFERENCES}

1. Darwin, C. (1859). On the Origin of Species by Means of Natural Selection, or the Preservation of Favoured Races in the Struggle for Life (John Murray).

2. Mayr, E. (1999). Systematics and the Origin of Species, from the Viewpoint of a Zoologist (Harvard University Press).

3. Ohta, T. (1973). Slightly deleterious mutant substitutions in evolution. Nature 246, 96-98.

4. Ohta, T. (1992). The nearly neutral theory of molecular evolution. Annu. Rev. Ecol. Syst. 23, 263-286.

5. Gossmann, T.I., Keightley, P.D., and Eyre-Walker, A. (2012). The effect of variation in the effective population size on the rate of adaptive molecular evolution in eukaryotes. Genome Biol. Evol. 4, 658-667.

6. Lanfear, R., Kokko, H., and Eyre-Walker, A. (2014). Population size and the rate of evolution. Trends Ecol. Evol. 29, 33-41.

7. Nam, K., Munch, K., Mailund, T., Nater, A., Greminger, M.P., Krützen, M., Marquès-Bonet, T., and Schierup, M.H. (2017). Evidence that the rate of strong selective sweeps increases with population size in the great apes. Proc. Natl. Acad. Sci. USA 114, 1613-1618.

8. Rousselle, M., Simion, P., Tilak, M.-K., Figuet, E., Nabholz, B., and Galtier, N. (2020). Is adaptation limited by mutation? A timescale-dependent effect of genetic diversity on the adaptive substitution rate in animals. PLoS Genet. 16, e1008668.

9. Lamichhaney, S., Berglund, J., Almén, M.S., Maqbool, K., Grabherr, M., Martinez-Barrio, A., Promerová, M., Rubin, C.-J., Wang, C., Zamani, N., et al. (2015). Evolution of Darwin's finches and their beaks revealed by genome sequencing. Nature 518, 371-375.

10. Burri, R., Nater, A., Kawakami, T., Mugal, C.F., Olason, P.I., Smeds, L., Suh, A., Dutoit, L., Bureš, S., Garamszegi, L.Z., et al. (2015). Linked selection and recombination rate variation drive the evolution of the genomic landscape of differentiation across the speciation continuum of Ficedula flycatchers. Genome Res. 25, 1656-1665.

11. Bourgeois, Y.X.C., Delahaie, B., Gautier, M., Lhuillier, E., Malé, P.G., Bertrand, J.A.M., Cornuault, J., Wakamatsu, K., Bouchez, O., Mould, C., et al. (2017). A novel locus on chromosome 1 underlies the evolution of a melanic plumage polymorphism in a wild songbird. R. Soc. Open Sci. 4, 160805.

12. Leroy, T., Anselmetti, Y., Tilak, M.-K., Bérard, S., Csukonyi, L., Gabrielli, M., Scornavacca, C., Milá, B., Thébaud, C., and Nabholz, B. (2019). A bird's white-eye view on neosex chromosome evolution. bioRxiv. https://doi.org/10.1101/505610.

13. Singhal, S., Leffler, E.M., Sannareddy, K., Turner, I., Venn, O., Hooper, D.M., Strand, A.I., Li, Q., Raney, B., Balakrishnan, C.N., et al. (2015). Stable recombination hotspots in birds. Science 350, 928-932.

14. Corcoran, P., Gossmann, T.I., Barton, H.J., Slate, J., and Zeng, K.; Great Tit HapMap Consortium (2017). Determinants of the efficacy of natural selection on coding and noncoding variability in two passerine species. Genome Biol. Evol. 9, 2987-3007.

15. Lundberg, M., Liedvogel, M., Larson, K., Sigeman, H., Grahn, M., Wright, A., Åkesson, S., and Bensch, S. (2017). Genetic differences between willow warbler migratory phenotypes are few and cluster in large haplotype blocks. Evol. Lett. 1, 155-168.

16. Jarvis, E.D., Mirarab, S., Aberer, A.J., Li, B., Houde, P., Li, C., Ho, S.Y.W., Faircloth, B.C., Nabholz, B., Howard, J.T., et al. (2014). Whole-genome analyses resolve early branches in the tree of life of modern birds. Science $346,1320-1331$
17. Backström, N., Forstmeier, W., Schielzeth, H., Mellenius, H., Nam, K., Bolund, E., Webster, M.T., Ost, T., Schneider, M., Kempenaers, B., and Ellegren, H. (2010). The recombination landscape of the zebra finch Taeniopygia guttata genome. Genome Res. 20, 485-495.

18. Kawakami, T., Smeds, L., Backström, N., Husby, A., Qvarnström, A., Mugal, C.F., Olason, P., and Ellegren, H. (2014). A high-density linkage map enables a second-generation collared flycatcher genome assembly and reveals the patterns of avian recombination rate variation and chromosomal evolution. Mol. Ecol. 23, 4035-4058.

19. Hill, W.G., and Robertson, A. (1966). The effect of linkage on limits to artificial selection. Genet. Res. 8, 269-294.

20. Rousselle, M., Laverré, A., Figuet, E., Nabholz, B., and Galtier, N. (2019). Influence of recombination and GC-biased gene conversion on the adaptive and nonadaptive substitution rate in mammals versus birds. Mol. Biol. Evol. 36, 458-471.

21. Galtier, N. (2016). Adaptive protein evolution in animals and the effective population size hypothesis. PLoS Genet. 12, e1005774.

22. Díez-Del-Molino, D., Sánchez-Barreiro, F., Barnes, I., Gilbert, M.T.P., and Dalén, L. (2018). Quantifying temporal genomic erosion in endangered species. Trends Ecol. Evol. 33, 176-185.

23. Peart, C.R., Tusso, S., Pophaly, S.D., Botero-Castro, F., Wu, C.-C., Aurioles-Gamboa, D., Baird, A.B., Bickham, J.W., Forcada, J., Galimberti, F., et al. (2020). Determinants of genetic variation across eco-evolutionary scales in pinnipeds. Nat. Ecol. Evol. 4, 1095-1104.

24. Loire, E., Chiari, Y., Bernard, A., Cahais, V., Romiguier, J., Nabholz, B., Lourenço, J.M., and Galtier, N. (2013). Population genomics of the endangered giant Galápagos tortoise. Genome Biol. 14, R136.

25. Rogers, R.L., and Slatkin, M. (2017). Excess of genomic defects in a woolly mammoth on Wrangel island. PLoS Genet. 13, e1006601.

26. Robinson, J.A., Brown, C., Kim, B.Y., Lohmueller, K.E., and Wayne, R.K. (2018). Purging of strongly deleterious mutations explains long-term persistence and absence of inbreeding depression in island foxes. Curr. Biol. 28, 3487-3494.e4.

27. Robinson, J.A., Ortega-Del Vecchyo, D., Fan, Z., Kim, B.Y., vonHoldt, B.M., Marsden, C.D., Lohmueller, K.E., and Wayne, R.K. (2016). Genomic flatlining in the endangered island fox. Curr. Biol. 26, 1183-1189.

28. Kutschera, V.E., Poelstra, J.W., Botero-Castro, F., Dussex, N., Gemmell, N.J., Hunt, G.R., Ritchie, M.G., Rutz, C., Wiberg, R.A.W., and Wolf, J.B.W. (2020). Purifying selection in corvids is less efficient on islands. Mol. Biol. Evol. 37, 469-474.

29. Hamabata, T., Kinoshita, G., Kurita, K., Cao, P.-L., Ito, M., Murata, J., Komaki, Y., Isagi, Y., and Makino, T. (2019). Endangered island endemic plants have vulnerable genomes. Commun. Biol. 2, 244.

30. Kimura, M. (1968). Evolutionary rate at the molecular level. Nature 217 , $624-626$.

31. King, J.L., and Jukes, T.H. (1969). Non-Darwinian evolution. Science 164, 788-798.

32. Chen, J., Glémin, S., and Lascoux, M. (2017). Genetic diversity and the efficacy of purifying selection across plant and animal species. Mol. Biol. Evol. 34, 1417-1428.

33. Plomion, C., Aury, J.-M., Amselem, J., Leroy, T., Murat, F., Duplessis, S., Faye, S., Francillonne, N., Labadie, K., Le Provost, G., et al. (2018). Oak genome reveals facets of long lifespan. Nat. Plants 4, 440-452.

34. Romiguier, J., Gayral, P., Ballenghien, M., Bernard, A., Cahais, V., Chenuil, A., Chiari, Y., Dernat, R., Duret, L., Faivre, N., et al. (2014). Comparative population genomics in animals uncovers the determinants of genetic diversity. Nature 515, 261-263.

35. Doyle, J.M., Hacking, C.C., Willoughby, J.R., Sundaram, M., and DeWoody, J.A. (2015). Mammalian genetic diversity as a function of habitat, body size, trophic class, and conservation status. J. Mammal. 96, 564-572.

36. Willoughby, J.R., Sundaram, M., Wijayawardena, B.K., Kimble, S.J.A., Ji, Y., Fernandez, N.B., Antonides, J.D., Lamb, M.C., Marra, N.J., and DeWoody, J.A. (2015). The reduction of genetic diversity in threatened 
vertebrates and new recommendations regarding IUCN conservation rankings. Biol. Conserv. 191, 495-503.

37. Brüniche-Olsen, A., Kellner, K.F., and DeWoody, J.A. (2019). Island area, body size and demographic history shape genomic diversity in Darwin's finches and related tanagers. Mol. Ecol. 28, 4914-4925.

38. Brüniche-Olsen, A., Kellner, K.F., Anderson, C.J., and DeWoody, J.A. (2018). Runs of homozygosity have utility in mammalian conservation and evolutionary studies. Conserv. Genet. 19, 1295-1307.

39. Kondrashov, A.S. (1988). Deleterious mutations and the evolution of sexual reproduction. Nature 336, 435-440.

40. Lynch, M., and Gabriel, W. (1990). Mutation load and the survival of small populations. Evolution 44, 1725-1737.

41. Warren, B.H., Hagen, O., Gerber, F., Thébaud, C., Paradis, E., and Conti, E. (2018). Evaluating alternative explanations for an association of extinction risk and evolutionary uniqueness in multiple insular lineages. Evolution 72, 2005-2024.

42. Valente, L., Phillimore, A.B., Melo, M., Warren, B.H., Clegg, S.M., Havenstein, K., Tiedemann, R., Illera, J.C., Thébaud, C., Aschenbach, T., and Etienne, R.S. (2020). A simple dynamic model explains the diversity of island birds worldwide. Nature 579, 92-96.

43. Chapman, J.A., Ho, I., Sunkara, S., Luo, S., Schroth, G.P., and Rokhsar, D.S. (2011). Meraculous: de novo genome assembly with short pairedend reads. PLoS ONE 6, e23501.

44. Putnam, N.H., O’Connell, B.L., Stites, J.C., Rice, B.J., Blanchette, M., Calef, R., Troll, C.J., Fields, A., Hartley, P.D., Sugnet, C.W., et al. (2016). Chromosome-scale shotgun assembly using an in vitro method for longrange linkage. Genome Res. 26, 342-350.

45. Bolger, A.M., Lohse, M., and Usadel, B. (2014). Trimmomatic: a flexible trimmer for Illumina sequence data. Bioinformatics 30, 2114-2120.

46. Li, H. (2013). Aligning sequence reads, clone sequences and assembly contigs with BWA-MEM. arXiv, arXiv, 1303.3997. https://arxiv.org/abs/ 1303.3997.

47. Broad Institute (2019). Picard Toolkit. http://broadinstitute.github.io/picard/.

48. McKenna, A., Hanna, M., Banks, E., Sivachenko, A., Cibulskis, K., Kernytsky, A., Garimella, K., Altshuler, D., Gabriel, S., Daly, M., and DePristo, M.A. (2010). The Genome Analysis Toolkit: a MapReduce framework for analyzing next-generation DNA sequencing data. Genome Res. 20, 1297-1303.

49. Allio, R., Schomaker-Bastos, A., Romiguier, J., Prosdocimi, F., Nabholz, B., and Delsuc, F. (2020). MitoFinder: Efficient automated large-scale extraction of mitogenomic data in target enrichment phylogenomics. Mol. Ecol. Resour. 20, 892-905.

50. Ranwez, V., Douzery, E.J.P., Cambon, C., Chantret, N., and Delsuc, F. (2018). MACSE v2: toolkit for the alignment of coding sequences accounting for frameshifts and stop codons. Mol. Biol. Evol. 35, 2582-2584.

51. Nguyen, L.-T., Schmidt, H.A., von Haeseler, A., and Minh, B.Q. (2015). IQTREE: a fast and effective stochastic algorithm for estimating maximumlikelihood phylogenies. Mol. Biol. Evol. 32, 268-274.

52. She, R., Chu, J.S.-C., Uyar, B., Wang, J., Wang, K., and Chen, N. (2011). genBlastG: using BLAST searches to build homologous gene models. Bioinformatics 27, 2141-2143.

53. Eddy, S.R. (2011). Accelerated profile HMM searches. PLoS Comput. Biol. 7, e1002195, e1002195.

54. Li, H., and Durbin, R. (2011). Inference of human population history from individual whole-genome sequences. Nature 475, 493-496.

55. R Core Team (2018). R: A Language and Environment for Statistical Computing (R Foundation for Statistical Computing).

56. Lamichhaney, S., Han, F., Berglund, J., Wang, C., Almén, M.S., Webster, M.T., Grant, B.R., Grant, P.R., and Andersson, L. (2016). A beak size locus in Darwin's finches facilitated character displacement during a drought. Science 352, 470-474.

57. Zink, R.M., and Vázquez-Miranda, H. (2019). Species limits and phylogenomic relationships of Darwin's finches remain unresolved: potential consequences of a volatile ecological setting. Syst. Biol. 68, 347-357.
58. Parker, P., Li, B., Li, H., and Wang, J. (2012). The genome of Darwin's finch (Geospiza fortis). Gigascience.

59. Ellegren, H., Smeds, L., Burri, R., Olason, P.I., Backström, N., Kawakami, T., Künstner, A., Mäkinen, H., Nadachowska-Brzyska, K., Qvarnström, A., et al. (2012). The genomic landscape of species divergence in Ficedula flycatchers. Nature 491, 756-760.

60. Warren, W.C., Clayton, D.F., Ellegren, H., Arnold, A.P., Hillier, L.W. Künstner, A., Searle, S., White, S., Vilella, A.J., Fairley, S., et al. (2010). The genome of a songbird. Nature 464, 757-762.

61. Laine, V.N., Gossmann, T.I., Schachtschneider, K.M., Garroway, C.J., Madsen, O., Verhoeven, K.J.F., de Jager, V., Megens, H.-J., Warren, W.C., Minx, P., et al.; Great Tit HapMap Consortium (2016). Evolutionary signals of selection on cognition from the great tit genome and methylome. Nat. Commun. 7, 10474

62. Recuerda, M., Vizueta, J., Cuevas-Caballé, C., Blanco, G., Rozas, J., and Milá, B. (2020). Chromosome-level genome assembly of the common chaffinch (Aves: Fringilla coelebs): a valuable resource for evolutionary biology. bioRxiv. https://doi.org/10.1101/2020.11.30.404061.

63. Scornavacca, C., Belkhir, K., Lopez, J., Dernat, R., Delsuc, F., Douzery, E.J.P., and Ranwez, V. (2019). OrthoMaM v10: scaling-up orthologous coding sequence and exon alignments with more than one hundred mammalian genomes. Mol. Biol. Evol. 36, 861-862.

64. Guéguen, L., Gaillard, S., Boussau, B., Gouy, M., Groussin, M., Rochette, N.C., Bigot, T., Fournier, D., Pouyet, F., Cahais, V., et al. (2013). Bio++: efficient extensible libraries and tools for computational molecular evolution. Mol. Biol. Evol. 30, 1745-1750.

65. Nadachowska-Brzyska, K., Burri, R., Olason, P.I., Kawakami, T., Smeds, L., and Ellegren, H. (2013). Demographic divergence history of pied flycatcher and collared flycatcher inferred from whole-genome resequencing data. PLoS Genet. 9, e1003942.

66. Kim, S., Cho, Y.S., Kim, H.-M., Chung, O., Kim, H., Jho, S., Seomun, H., Kim, J., Bang, W.Y., Kim, C., et al. (2016). Comparison of carnivore, omnivore, and herbivore mammalian genomes with a new leopard assembly. Genome Biol. 17, 211.

67. Smeds, L., Qvarnström, A., and Ellegren, H. (2016). Direct estimate of the rate of germline mutation in a bird. Genome Res. 26, 1211-1218.

68. Brommer, J.E., Gustafsson, L., Pietiäinen, H., and Merilä, J. (2004). Singlegeneration estimates of individual fitness as proxies for long-term genetic contribution. Am. Nat. 163, 505-517.

69. Wickham, H. (2016). ggplot2: Elegant Graphics for Data Analysis (Springer-Verlag).

70. Wilke, C. (2016). cowplot: Streamlined Plot Theme and Plot Annotations for "ggplot2".

71. Bolívar, P., Mugal, C.F., Nater, A., and Ellegren, H. (2016). Recombination rate variation modulates gene sequence evolution mainly via GC-biased gene conversion, not Hill-Robertson interference, in an avian system. Mol. Biol. Evol. 33, 216-227.

72. Eyre-Walker, A., and Keightley, P.D. (2009). Estimating the rate of adaptive molecular evolution in the presence of slightly deleterious mutations and population size change. Mol. Biol. Evol. 26, 2097-2108.

73. Eyre-Walker, A., Woolfit, M., and Phelps, T. (2006). The distribution of fitness effects of new deleterious amino acid mutations in humans. Genetics 173, 891-900.

74. Pinheiro, J., Bates, D., DebRoy, S., and Sarkar, D.; R Core Team (2020). nlme: linear and nonlinear mixed effects models. https://CRAN R-project.org/package $=$ nlme.

75. Paradis, E., and Schliep, K. (2019). ape 5.0: an environment for modern phylogenetics and evolutionary analyses in R. Bioinformatics 35, 526-528.

76. Kassambara, A. (2018). ggplot2 (Based Publication Ready Plots).

77. Slowikowski, K. (2019). ggrepel: automatically position non-overlapping text labels with "ggplot2".

78. Yu, G., Smith, D.K., Zhu, H., Guan, Y., and Lam, T.T.-Y. (2017). ggtree: an r package for visualization and annotation of phylogenetic trees with their covariates and other associated data. Methods Ecol. Evol. 8, 28-36. 


\section{Report}

\section{STAR $\star M E T H O D S$}

\section{KEY RESOURCES TABLE}

\begin{tabular}{|c|c|c|}
\hline REAGENT or RESOURCE & SOURCE & IDENTIFIER \\
\hline \multicolumn{3}{|l|}{ Biological samples } \\
\hline 18 Zosterops borbonicus samples & Field sampling in Reunion, France & See Table S3 \\
\hline 15 Zosterops olivaceus samples & Field sampling in Reunion, France & See Table S3 \\
\hline 9 Zosterops mauritinaus samples & Field sampling in Mauritius & See Table S3 \\
\hline 1 Zosterops pallidus sample & Field sampling in South Africa & See Table S3 \\
\hline 11 Zosterops virenssamples & Field sampling in South Africa & See Table S3 \\
\hline 9 continental Fringilla coelebs samples & Field sampling in continental Spain & See Table S3 \\
\hline 15 Fringilla coelebs palmae samples & Field sampling in La Palma, Spain & See Table S3 \\
\hline 10 Fringilla teydae samples & Field sampling in Tenerife, Spain & See Table S3 \\
\hline \multicolumn{3}{|l|}{ Deposited data } \\
\hline Raw reads Taeniopygia \& Poephila & 13 & BioProject PRJEB10586 \\
\hline Raw reads Parus & 14 & BioProject PRJNA381923 \\
\hline Raw reads Phylloscopus & 15 & BioProject PRJNA319295 \\
\hline Raw reads Darwin's finches & 9 & BioProject PRJNA263122 \\
\hline Raw reads Ficedula & 10 & BioProject PRJEB7359 \\
\hline Raw reads Zosterops & 11,12 and this study & BioProjects PRJEB18566, PRJNA530916, PRJNA661201 \\
\hline Raw reads Fringilla & This study & BioProject PRJNA661201 \\
\hline \multicolumn{3}{|l|}{ Software and algorithms } \\
\hline Meraculous v. 2.2.2.5 & 43 & https://jgi.doe.gov/data-and-tools/meraculous/ \\
\hline HiRise & 44 & $\begin{array}{l}\text { https://github.com/DovetailGenomics/HiRise_ } \\
\text { July2015_GR }\end{array}$ \\
\hline Trimmomatic & 45 & http://www.usadellab.org/cms/?page=trimmomatic \\
\hline BWA mem & 46 & http://bio-bwa.sourceforge.net/ \\
\hline Picard v. 1.140 & 47 & http://broadinstitute.github.io/picard/ \\
\hline GATK v. 3.7 & 48 & https://gatk.broadinstitute.org/hc/en-us \\
\hline MitoFinder v.1.1 & 49 & https://github.com/RemiAllio/MitoFinder \\
\hline Macse v.2 & 50 & $\begin{array}{l}\text { https://bioweb.supagro.inra.fr/macse/ } \\
\text { index.php?menu=releases }\end{array}$ \\
\hline IQTREE & 51 & http://www.iqtree.org/ \\
\hline genBlastG & 52 & http://genome.sfu.ca/genblast/download.html \\
\hline HMMER toolkit & 53 & http://hmmer.org/ \\
\hline PSMC & 54 & https://github.com/lh3/psmc \\
\hline Grapes. v1.0 & 21 & https://github.com/BioPP/grapes \\
\hline R v3.6.3 & 55 & https://cran.r-project.org/ \\
\hline Scripts used & This study & https://osf.io/uw6mb/ \\
\hline
\end{tabular}

\section{RESOURCE AVAILABILITY}

\section{Lead contact}

Further information and requests for resources and reagents should be directed to and will be fulfilled by the Lead Contact, Benoit Nabholz (benoit.nabholz@umontpellier.fr).

Materials availability

This study did not generate new unique reagents.

Data and code availability

All raw sequencing data have been deposited in the Sequence Read Archive (SRA) under BioProject:PRJNA661201. All scripts and programs used are available at the following Open Science Framework repository: https://osf.io/uw6mb/ 


\section{EXPERIMENTAL MODEL AND SUBJECT DETAILS}

Species included in the study

In this study, we both reanalyzed publicly available data and generated our own sequencing data from 25 passerine species (Table S3). By generating new sequencing data, our objective was to target taxa containing both island and continental relatives (chaffinches and white-eyes) in order to increase our statistical power. More broadly, our comparison is only based on species with relatively similar body-mass, longevity and clutch-size. This control was introduced to reduce the risk of some confounding factors that could correlate with $\mathrm{Ne}^{34}$ in order to be able to truly assess the effect of insularity.

Species distribution and IUCN red list status

Species range sizes were obtained from BirdLife (http://datazone. birdlife.org/home) or estimated based on the information shown on the IUCN-red list webpage using CalcMaps (https://www.calcmaps.com/map-area/). For endemic island species, we considered the total island area as a maximum bound for the population range. The IUCN red list conservation status is given at the species level and not at below-species level. As a consequence, we either considered this information to be missing for both populations (e.g., Certhidea fusca E and C. fusca L) or we only used the status for the most widely distributed species (e.g., the Least Concern (LC) status for the population with a large continental distribution rather than for the island one as in F. coelebs palmae). For Ficedula speculigera, a species with $\mathrm{D}_{\mathrm{A}}>0.002$ (Methods $S 1$ ) and recognized as a distinct species from $F$. hypoleuca, no information is yet available in the IUCN red list database.

\section{METHOD DETAILS}

DNA extraction and sequencing (Zosterops and Fringilla species)

All Zosterops and Fringilla individuals were captured using mist nets. With the exception of African Zosterops species (Z. pallidus and $Z$. virens, see below), we collected blood samples for each bird by venipuncture of the brachial vein and stored blood in absolute ethanol at $-20^{\circ} \mathrm{C}$ until DNA extraction. For African species, $Z$. pallidus and Z. virens individuals, DNA was extracted from liver, muscle or blood. For these samples, voucher specimens are stored at the Museum National d'Histoire Naturelle (MNHN), Paris, France and a tissue duplicate is deposited in the National Museum Bloemfontein (South Africa). For all Zosterops and Fringilla samples, total genomic DNA was extracted using the DNeasy Blood and Tissue kit (QIAGEN, Valencia, CA) following the manufacturer's instructions. Library preparation (1.0 $\mu \mathrm{g}$ DNA used per sample) and Illumina high-throughput sequencing using a paired-end 150 bp (PE150) strategy were performed at Novogene (Cambridge, UK) to a minimum sequencing yield of $18 \mathrm{~Gb}$ per sample (i.e., $~ 15 X$ coverage). Details on samples are available in Table S3. For these species, we used exactly the same approach as for the publicly available data for variant identification and sequence reconstruction strategies, as described in Methods S1. All newly sequenced raw reads are available under the SRA BioProject : PRJNA661201.

\section{Publicly available sequencing data}

We collected publicly available raw sequencing data on SRA from a large range of studies (Table S3). The phylogenetic relationships among Darwin's finches are not fully resolved, ${ }^{9,56,57}$ so we first evaluate the net divergence between all pairs of species to delimit 9 groups of species with a net divergence $\left(D_{A}>0.1 \%\right.$; Methods $\left.S 1\right)$. Within each group, we selected a single population based on the number of sequenced individuals that were publicly available. ${ }^{9}$ Variant identification and sequence reconstruction steps are described in Methods S1.

\section{Variant identification}

We used Trimmomatic $\left(v .0 .33^{45}\right)$ to remove adapters, stringently trim and filter reads using the following set of parameters: LEADING:3 TRAILING:3 SLIDINGWINDOW:4:15 MINLEN:50. All trimmed reads were then mapped against the reference genome for each clade (see above) with BWA mem (v. 0.7.12 ${ }^{46}$ ) using default settings. Unmapped reads and mapped reads with a quality (MQ) below 20 were then discarded. Potential PCR duplicates were then flagged using MarkDuplicates v. 1.140 (Picard tools ${ }^{47}$ ). Variant calling was then performed using GATK (v. 3.7 ${ }^{48}$ ). First, we used HaplotypeCaller on single samples (gVCF) to call SNPs using default parameters. For each species, we then performed a joint genotyping ("GenotypeGVCFs"). To ensure high quality in our dataset, we filtered out lowquality SNPs using several settings: a quality by depth (QD) $<2.0$, a Fisher Strand (FS) bias $>60$, a mapping quality (MQ) $<40$, a MQranksum $<-2$ or a ReadPosRankSum $<-2$ or a Raw Mapping Quality (Raw_MQ) $<45,000$. SNPs satisfying one or more of these conditions were discarded. For every group of species, we performed principal component analyses (PCA) based on a random sampling of SNPs over the genome (50-200k) to capture additional levels of population structure or an unfortunate misnaming of an individual that could have occurred at some point between the bird sampling campaign and the analysis of the raw sequencing data.

\section{QUANTIFICATION AND STATISTICAL ANALYSIS}

\section{Gene models \& orthology prediction}

We used one reference genome for all species belonging to the same clade (Table S3). We used the genome and gene models of a medium ground-finch individual (Geospiza fortis; assembly GeoFor_1.0; GCF_000277835 ${ }^{58}$ ) for all Darwin's finches, a collared 
flycatcher (Ficedula albicollis, GCF_000247815; assembly FicAlb_1.4 ${ }^{59}$ ) for all Ficedula, a zebra finch (Taeniopygia guttata; GCF_000151805; assembly taeGut3.2.460) for the Estrildidae, a Reunion grey white-eye (Zosterops borbonicus; GCA_007252995; assembly ZoBo_15179_v2.0 ${ }^{12}$ ) for all Zosterops. We also used the genome of the willow warbler (Phylloscopus trochilus; GCA_002305835; assembly ASM230583v1 ${ }^{15}$ ) and the great tit (Parus major; GCF_001522545.2; assembly Parus_major1.1 ${ }^{61}$ ). For all the investigated chaffinches, we used a newly generated assembly of Fringilla coelebs (Methods S1, version "HiRise" of Recuerda et al. $\left.{ }^{62}\right)$. For this latter species, as well as for the willow warbler ( $P$. trochilus), no gene models were available and we therefore first performed a protein homology detection and intron resolution using genBlastG ${ }^{52}$ (http://genome.sfu.ca/genblast/download.html) with the following options "-p genblastg -c 0.8 -r 3.0 -gff -e 1e-10."

To analyze the same orthologous sequences in all species, we used the set of 8253 orthologs identified by Jarvis et al. ${ }^{16}$ (http:// gigadb.org/dataset/101041). Then, we added the sequence of our species to this set of orthogroups using the method described in Scornavacca et al. ${ }^{63}$ Briefly, each orthogroup was used to build an HMM profile using the HMMER toolkit. ${ }^{53}$ Then, for each new sequence, hmmscan was used on the HMM database to get the best hits among the orthogroups. For each orthogroup, the most similar sequences for each species were then detected via hmmsearch. Outputs from hmmsearch and hmmscan were considered to be accurate if the first hit score was substantially better than the second best one (in order to limit the risk of paralogy), following a best-reciprocal-hit approach when the results of both programs were compared. ${ }^{63}$

\section{Effective population size estimates}

Historical demographic variations in Ne were estimated using the Pairwise Sequentially Markovian Coalescent (PSMC) model implemented in the software PSMC. ${ }^{48}$ Fasta sequences were converted to the PSMC fasta format using a C++ program (Fasta2PSMCFasta: https://osf.io/uw6mb/) written using BIO++ library. ${ }^{64}$ Only scaffolds longer than $500 \mathrm{~Kb}$ were considered. We used block length of $100 \mathrm{bp}$, with no more than $20 \%$ of missing data per block, as implemented in "fq2psmcfa" (https://github.com/lh3/psmc).

For each species, PSMC analyses were run using two randomly selected individuals. To identify suitable parameters, several -t and - $p$ parameters were tested including - $p$ " $4+30^{\star} 2+4+6+10^{\prime \prime}$ (as in Nadachowska-Brzyska et al. ${ }^{65}$ ) and $-p$ " $4+25^{\star} 2+4+6$ " (as in Kim et al. ${ }^{66}$ ) but also $-p$ " $4+10 * 3+4$ " and $-p$ " $5^{\star} 1+25^{\star} 2+6$." The best combination (t15 - r4 - $p$ " $5 * 1+25^{\star} 2+6$ ") was manually chosen after excluding other parameter values leading to large differences between the two individuals from the same species. Then, we randomly selected one individual and excluded the first four atomic time intervals to exclude the noisy estimates generally generated by PSMC for very recent times and therefore strengthen the reliability of the average estimates of $\mathrm{Ne}$ over the last million years.

Time was scaled assuming a mutation rate of $4.6 \times 10^{-9}$ mutation/site/generation as estimated ${ }^{67}$ and a generation time of 2 years. ${ }^{65,68}$ Results were plotted in R (v3.6.3 ${ }^{55}$ ) using the function "psmc.results" [69; http://datadryad.org/resource/doi:10. $5061 /$ dryad.0618v/4] and with the $R$ packages ggplot $2^{69}$ and cowplot. ${ }^{70}$

\section{Summary statistics of the polymorphic data}

$\pi_{S}$ and $\pi_{N} / \pi_{S}$ ratios were computed using seq_stat_coding from reconstructed fasta sequences (Methods S1) using a publicly available Bio++ script and a procedure previously described ${ }^{42}$ (https://osf.io/uw6mb/). We empirically validated that our $\pi_{\mathrm{S}}$ and $\pi_{\mathrm{N}} / \pi_{\mathrm{S}}$ estimates were not impacted by the variable number of samples per species. In addition, we used the $\pi_{N} / \pi_{S}$ estimates based on the site frequency spectra at both non-synonymous and synonymous sites as described in Rousselle et al. ${ }^{18}$ to check the accuracy of these estimates (see also Table S2). Guanine-Cytosine (GC) content at third-codon positions of protein-coding genes (hereafter GC3), an excellent proxy of the local recombination rate in birds ${ }^{71}$ was also computed under seq_stat_coding. To estimate the withingenome variation in the efficacy of selection, we estimated $\pi_{N} / \pi_{S}$ on sets of genes representing a total concatenated coding alignment of $2 \mathrm{Mb}$, after sorting genes by ascending values of GC3. The last window corresponding to genes exhibiting the highest GC3 values was only considered if this window contained at least $1 \mathrm{Mb}$ of coding sequence.

\section{Summary statistics of the divergence data}

We used the method implemented by Galtier ${ }^{21}$ (Grapes. v1.0) to estimate $\alpha, \omega_{\mathrm{A}}$ and $\omega_{\mathrm{NA}}$ using the approach introduced by EyreWalker \& Keightley. ${ }^{72}$ Briefly, we fitted both a negative Gamma distribution and an exponential distribution to the synonymous and non-synonymous Site Frequency Spectrum (SFS) (the so-called GammaExpo model ${ }^{21}$ ) to model the distribution of fitness effect (DFE). Fitted parameters of the DFE were then used to compute the expected $d_{N} / d_{S}$ under near neutrality (i.e., without adaptive substitutions but including weakly deleterious substitutions), which was compared to the observed $d_{N} / d_{S}$ to estimate the adaptive substitution rate $\left(\omega_{A}\right)$ and the proportion of adaptive substitutions $(\alpha)\left[\right.$ with $\left.\omega_{A}=\alpha\left(d_{N} / d_{S}\right) \& \omega_{N A}=(1-\alpha)\left(d_{N} / d_{S}\right)\right]$. Potential recent changes in population size that affect the SFS were taken into account via the use of nuisance parameters capturing distortions of the SFS optimized alongside the DFE parameters. ${ }^{73}$

\section{Statistical analyses}

All statistical analyses were performed using R. ${ }^{55}$ We only considered models with a similar number of observations and compared these models based on the Akaike information criterion with a correction for small sample sizes (AICc). To test for the influence of the explanatory variables on $\pi_{S}$ and $\pi_{N} / \pi_{S}$, we used Phylogenetic Generalized Least Square (PGLS) models. Explanatory variables were always $\log _{10}$-transformed as this violated less frequently the assumption of normality, heteroscedasticity and independence of the residuals using a simple linear model. For PGLS, we used the model implemented in the "nlme" package. ${ }^{74}$ The mitochondrial phylogeny was considered as the species tree (Figure $\mathrm{S} 1$, newick file is available at https://osf.io/uw6mb/) taken into account assuming a 
Brownian correlation structure (using "corBrownian" from the "ape" package ${ }^{75}$ ). P value and AICc were computed using the anova.gls function. The rationale of the phylogenetic control is to account for the shared polymorphisms (part of species similarity that is explained by the inheritance from a common ancestor). The level of polymorphism of a given species is dynamically controlled by drift, mutation rate and natural selection. As soon as two species do not share a significant fraction of their polymorphism, there is no need to account for their phylogenetic proximity because their polymorphisms evolved independently. Therefore, the results of all the tests including with and without phylogenetic controls, transformed and untransformed $\pi_{S}$ and $\pi_{N} / \pi_{S}$ are presented in Table S1.

R plots were generated using a series of R packages: cowplot, ${ }^{70}$ ggplot $2,{ }^{69}$ ggpubr, ${ }^{76}$ ggrepel $^{77}$ and ggtree. $^{78}$ 